\title{
Internal Quality Assurance of Institutional Learning Outcomes: United States Case Study and Implications for Vietnam Higher Education
}

\section{Nhung Tuyet Thi Pham ${ }^{+}$, Binh Thanh Thi Nguyen}

\author{
Article history \\ Received: 20 April 2021 \\ Accepted: 02 May 2021 \\ Published: 30 June 2021

\section{Keywords \\ Internal quality assurance (IQA), institutional learning outcomes, achievements, challenges, quality} \\ improvement, accountability
}

\author{
University of Foreign Languages - Hue University, Vietnam \\ ${ }^{+}$Corresponding author • Email: pttnhung.hufl@hueuni.edu.vn
}

\begin{abstract}
Higher education institutions (HEIs) are under pressure to provide evidence of student success. In addition to traditional performance indicators such as GPA, grades, and rates of retention, graduation and employment, stakeholders also required institutions to provide evidence of institutional learning outcomes (ILOs). ILOs encompass the knowledge and skills that all students regardless of disciplines from a specific university are expected to demonstrate upon their graduation. This case study examines a complete internal quality assurance (IQA) cycle from a U.S. comprehensive university, including the adaptation of national authentic assessment measures, the use of technology in data analysis, the best practices to communicate assessment results to multiple internal stakeholders to facilitate leadership decisionmaking, the challenges encountered, and the improvement plans to sustain the procedure. Researchers also make recommendations to Vietnam HEIs that want to adopt IQA of ILOs for both quality improvement and accountability purposes.
\end{abstract}

\section{INTRODUCTION}

Over the past several years, various individuals, organizations, and legislators have continued to express concerns about the quality of higher education in the United States. Those concerns have triggered legislation and requirements at the federal and state levels and by regional accreditors to assess and report on student learning (Bassis, 2015; Jones, 2009; Nelson, 2014). Therefore, U.S. colleges and universities have been asked to provide increasing evidence of transparency and accountability. Providers are held responsible for their performance through the disclosure of comparative results of programs, educational processes, and outcomes to better inform prospective students and other stakeholders (Liu, 2011; McCormick, 2010). In 2005, the Spellings Commission report suggested that institutions be required to provide evidence of student learning outcomes (SLOs) for accountability (U.S. Department of Education, 2006). This report served as a push for revisiting outcomes assessment to demonstrate student success during their undergraduate experience. The interests of external stakeholders played an important role for higher education institutions (HEIs).

As a result, most regional accreditation agencies in U.S. have embedded the evidence of student learning outcomes in the accreditation standards. In an effort to demonstrate the evidence to the regional accreditation agencies, most HEIs set up internal quality assurance (IQA) process to assess the institutional learning outcomes (ILOs). ILOs are the knowledge and skills students achieve during the first two years of their undergraduate program. This often refers to the knowledge and skills acquired through a general education (GE) program. Since the courses in the GE program are taught by multiple faculty members who specialize in different disciplines across many colleges at the same time, the IQA process and assessment of ILOs are significantly different from assessments of academic programs. A thorough case study of one U.S. university's IQA process can be useful for other HEIs to learn from. This research study examines the IQA process of ILOs, the challenges encountered along the way, and the use of results to ensure continuous quality improvement.

Quality assurance has been implemented in most higher education systems across the globe in the past few decades. It is increasingly evident that external quality assurance (EQA) and internal quality assurance (IQA) play 
important roles in monitoring, managing, and enhancing the quality of teaching and learning. In Vietnamese context, the earliest signs of IQA implementation emerged nearly 20 years ago. At present, almost every university in Vietnam has established a unit specializing in QA but the development of concept is still in its infancy in most HEIs. Even in those universities with the more advanced processes, IQA is not explicit in defining strategic goals, including how to achieve them, how to analyze data, and how to encourage and motivate internal stakeholders to sustain their commitment to the process. Quality culture has been far more to be built and supported in Vietnamese HEIs (Pham, 2013). Due to its infancy in Vietnam HEIs, there is limited research regarding the IQA of ILOs. A U.S. case study offers guidance on how Vietnam HE might initiate IQA of ILOs.

\section{LITERATURE REVIEW}

\subsection{Research about ILOs and U.S. HEIs}

Over the past several years, various individuals, organizations, and legislators have continued to express concerns about the quality of higher education. Those concerns have triggered legislation and requirements at the federal and state levels and new policies from regional accreditors on how to assess and report on student learning (Bassis, 2015; Jones, 2009; Nelson, 2014). Regional accrediting organizations that are recognized by the Council for Higher Education Accreditation (CHEA) all include requirements for institutions related to assessing SLOs. Those requirements emphasize both articulating the outcomes and measuring and documenting student success (CHEA, n.d.).

One of the first comprehensive IQAs of ILOs took place in the late 1920s (Penn, 2011). Major ILO assessment initiatives were undertaken in the mid- 1980s and early 1990s, in response to demands that universities provide detailed, comprehensive IQAs of ILOs but in complying with all the compliance requirements, universities could easily lose focus on the core purpose of quality assurance: universities can improve student learning and performance of ILOs. Fletcher et al. (2012) stated that universities conduct ILOs to provide information about student learning, student progress, teaching quality, and program and institutional accountability. More recently, assessment methods for ILOs have focused on $21^{\text {st }}$ century skills. The four major approaches to gathering data and assessing these skills are (1) Embedding IQA process in GE programs; (2) Examining the psychometric component of the assessment measures, (3) Designing IQA approach to provide evidence of learning gains or the "value-added" of students' competency, and (4) Using multiple assessment measures to provide triangulated evidence of ILOs. Most of the four assessment approaches use data from standardized exams to provide evidence of students competency in $21^{\text {st }}$ century skills.

Beauchman and Waldenberger (2017) assessed a five-year IQA plan by using a course-embedded approach and then comparing student performance across majors to facilitate a discussion with committees and departments. AlLail and Oudghiri (2016) not only used a rubric to assess ILOs but also triangulated data with indirect measures, including a market study to learn about the institution's reputation, a student survey, and an employer survey. Martins et al. (2019) shared the ILOs results from two measures - the Valid Assessment of Learning in Undergraduate Education (VALUE) rubric and the National Survey of Student Engagement (NSSE). Notably, all of these researchers used VALUE rubric and NSSE to provide descriptive evidence of student learning for accountability purposes. The VALUE rubric, as proposed by American Association of Colleges and Universities (AAC\&U), has a set of 16 learning outcomes (2015) to assess the common ILOs. VALUE rubrics serve as a supportive measure of authentic assessment of students' application of knowledge to address real-life problems (Boyles, 2012). In addition to descriptive statistics of student learning, Eisnaugle (2018) and Rear (2019) looked for additional factors that would have a relationship to student learning. Eisnaugle's studies indicated a significant correlation between course assignment and course GPA, while Rear (2019) examined the relationship of GPA, admission and major on student learning.

\subsection{IQA process of ILOs assessment}

AAC\&U's (VALUE) project and rubrics have been implemented by many universities. The VALUE rubrics were developed as part of AAC\&U's Liberal Education and America's Promise (LEAP) initiative. One advantage of implementing VALUE rubrics is that data and studies such as the Multi-State Collaborative to Advance Quality Student Learning (MSC) and the Great Lakes College Association Project to Advance Learning, report their findings and share lessons learned through their IQA implementation. McConnell and Rhodes (2017) provided detailed ILOs information based on a large number of institutions. The VALUE rubrics were piloted and used by a diverse range of post-secondary education institutions including community colleges, regional comprehensives, and research institutions. These data sets allow institutions to benchmark student performance against peer universities. Brown et al. (2018) found that HEIs typically function in an autonomous and siloed culture when implementing changes, with various programs and offices operating independently of one another. This approach creates a challenge for those 
seeking to establish a holistic, institution wide assessment. The research suggests that a cohesive framework and cooperation across campus are critical for effective implementation of ILOs assessment.

\subsection{IQA process in Vietnam HEIs}

IQA is a function of Vietnam's HE quality assurance system (VNHEQAS), a framework developed within the AsiaPacific region (Asia-Pacific Quality Network, 2008). According to MOET policy, all higher education institutions in Vietnam must set up QA units or centers (MOET, 2007) with the intent of promoting activities that will assure quality within individual HEIs. Centers staffed by QA specialists have been formed at most Vietnamese universities. These centers are considered IQA units (Pham, 2013). To implement the IQA activities, Vietnamese HEIs have adopted diverse approaches and models. Some HEIs adopted a tailor-made quality approach that derives from institutional goals and embed from institutional culture to fulfill internal and external requirements. This approach is mostly driven by accreditation requirements, therefore, the IQA system is built based on the PDCA Deming cycle with four basic elements of institutional goals, monitoring instruments, evaluation instruments, and improvement of quality. The second IQA approach is to use the ISO model to arrange and manage their documentation systems more effectively. The third approach is to embed the MOET's standards into their quality management guide (Nguyen, 2012).

The IQA infrastructure has been in place in Vietnam HEIs for almost 10 years. IQA research has been conducted to meet the programmatic accreditation for education programs (Van \& Phan, 2020), to share experience to implement IQA system at a specific HEI in Vietnam (Vo \& Ngo, 2020), on the use of technology for IQA system (Nguyen, 2020) or for suggestions on how to improve the IQA system in general (Le, 2020). However, there are limited research studies addressing IQA implementation for the assessment of ILOs. This research study provides some implications for Vietnam HEIs to implement IQA for ILOs to demonstrate evidence of quality student learning to internal and external stakeholders.

\section{MATERIALS AND METHODS}

\subsection{Context}

The IQA process in our study involves a regional comprehensive university in the Midwest of United States that had recently revised its goals for its GE program. The case study was chosen because it had just completed a full cycle of IQA process for ILOs and therefore could provide insights for other HEIs to improve the IQA process.

Its Carnegie classification is Comprehensive Universities, which offer undergraduate and graduate programs. Enrollment is just over 12,000 undergraduate and graduate students. According to university faculty, the university's previous GE program was a collection of unconnected courses that operated in a siloed culture, particularly in the context of implementing changes related to QA (Brown et al., 2018). In addition, the previous GE program focused on input measures in the form of courses and their specific competencies, and not on outcome measures (Bruce, 2018). The university sought to develop a framework to implement a more holistic IQA approach which would allow it to assess the impact of ILOs across multiple disciplines and colleges. The mission of the GE program has always been to provide students with foundational knowledge and skills that encompasses all baccalaureate programs. Under the revised GE program, courses must show how they align with and meet the specific outcomes for the university ILOs. The IQA process was designed so that faculty can submit courses to the faculty senate committee for consideration of inclusion in the GE program. As part of that submission, faculty must include information on how they will meet and assess the prescribed ILOs. Courses are also reviewed by a GE committee for recertification and to ensure they are following the IQA plan and that student artifacts align with desired ILOs.

\subsection{Study Purpose and Research Questions}

This research used case study to provide a rich description of the phenomenon (Yin, 1994). A case can be a person, a small group, a program, or an institution. As stated by Merriam (1998), a case study provides an in-depth description of a single instance, phenomenon, or social unit. Creswell (2014) also stated that a case has a clear boundary and can provide an in-depth comprehension of the case. This case study looks in-depth at procedures of IQA for ILOs assessment. We examined the revised procedures in order to address the following questions:

1. What was the IQA process of ILOs assessment?

2. What challenges did the university encounter?

3. How were the assessment results used for continuous improvement?

\subsection{Data Collection and Analysis}

The alignment of several components of the GE courses, IQA process, and data collection was very intentional. The goal was to ensure courses maintain alignment with the competencies and that faculty can collect and report data with minimal additional workload. Any GE courses going through the recertification process need to demonstrate that 
the learning outcomes and assignments align with a specific ILOs' competency. This ensures courses continue to align with the ILOs' competencies and goals. For courses aligned to a skill-based competency, students' artifacts from one assignment were required. Faculty chose an assignment that meets all the dimensions in the modified VALUE rubric for university data collection. The intent is for faculty to use an existing or typical assignment that was already being implemented in their course rather than create a new assignment for the ILOs assessment because this more authentic assessment does not create much additional work for faculty. Because assessment is embedded within all sections of the courses and is evaluated by the faculty member teaching each section, the process is streamlined.

For this study, the university collected students' artifacts from 230 course sections aligned with Competency 1 (written communication), Competency 2 (oral communication), Competency 3 (quantitative literacy) and Competency 5 (managing information). Nearly 5,000 artifacts were analyzed. The major analysis was descriptive analysis, with a focus on the average of each component in the competency rubric to determine where students performed well and where they needed more support. The data collection and data analysis from an assessment management system (AMS) also led to a more streamlined ILOs assessment process. The first advantage was that the technology was integrated with the existing learning management system (LMS) and enabled a relatively automated transfer of information into the AMS. Therefore, faculty utilized and graded the students' artifacts using the LMS. As most faculty were familiar with LMS, this also helped to encourage their participation. The second advantage of using technology was that all data were loaded directly into the AMS and accessible only by people with specific privileges, thereby protecting student confidentiality. The third advantage of technology, was efficiency (e.g., time savings) in the data analysis, as the AMS could run various reports. Consequently, the university could collect a large sample of students' artifacts across multiple ILOs' competencies in a year. This comprehensive data collection enabled the university to capture a more accurate and complete picture of student learning and facilitated actions for improvement based on ILOs assessment results in the later step. The fourth advantage for data collection was that the technology provided faculty and the institution with individualized assessment reports.

\section{RESULTS AND DISCUSSION \\ 4.1. Results}

\subsubsection{IQA process of ILOs assessment}

The university IQA system follows six phases of the IQA cycle: (1) plan and identify outcomes, (2) collect data, (3) analyze data, (4) share results, (5) identify and implement changes, and (6) assess impact of change (Kuh et al., 2015). The revised ILOs served student needs and the public interest by ensuring that students have strong foundational skills through providing a broad, enriched academic experience that both complements and supports their study within specialized disciplines. To capture the student learning of the ILOs competencies (10 in all), the university has used three major assessment measures: The General Education Assessment (GEA) Exam, the Modified VALUE rubrics, and the National Survey of Student Engagement (NSSE). The GEA and Modified VALUE rubrics serve as direct assessment measures of student learning outcomes and the NSSE serves as an indirect assessment measure of student learning outcomes. This paper discusses only the direct ILOs assessment measuremodified VALUE rubric. See Figure 1 for the IQA process.

In an effort to determine whether the teaching of the GE courses met the requirement of the university ILOs' competencies, the university first developed an IQA plan and timeline for data collection. In 2015-2016, the university conducted a series of planning meetings with faculty teaching in the GE program to collectively define the process for data collection. In the Fall 2016 semester,

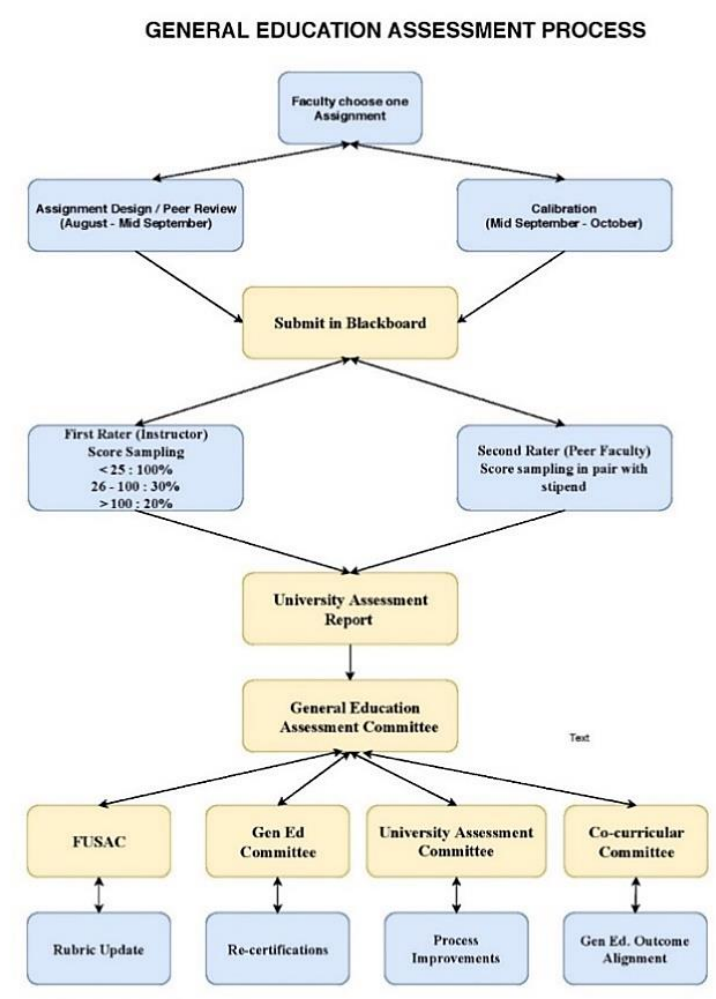

Figure 1. Midwest University IQA process of ILOs 
the institution provided face-to-face as well as online training for all instructors on how to use the modified rubrics. It was determined that pilot data would be collected in the Spring of 2017 semester and that student artifacts for five competencies (written communication, oral communication, quantitative literacy, critical/creative thinking, and managing information) would be collected. As this was the first time the university conducted an institution-wide ILOs assessment, instructors of all courses that aligned to a specific ILOs competency were asked to voluntarily provide students' artifacts for ILOs assessment. Data from four competencies (oral communication, quantitative literacy, creative/critical thinking, and managing information) were gathered in an Excel template and the written communication competency was collected through an AMS. The purpose of this pilot was to ensure the IQA process was appropriate before collecting artifacts of the five competencies from all courses.

* Two-Year Timeline

The data collection pilot was successful, therefore, from 2017-2018, the university implemented a two-year ILOs assessment plan for general education program (Table 1), using the course-embedded assessment (CBA) function in the AMS. Data were collected during the Fall semester, and in the Spring semester, the results and opportunities for teaching and learning improvement were discussed and documented.

Table 1. Two-Year ILOs Assessment Timeline

\begin{tabular}{|c|c|c|c|c|}
\hline \multirow{2}{*}{ Assessment and Evaluation Activity } & \multicolumn{2}{|c|}{$2017-2018$} & \multicolumn{2}{|c|}{$2018-2019$} \\
\hline & Fall & Spring & Fall & Spring \\
\hline Collect data/Evaluate data including the processes & $\begin{array}{c}\text { Competency } \\
1,2,3,5\end{array}$ & & $\begin{array}{c}\text { Competency } \\
4\end{array}$ & \\
\hline Deliver report findings to constituents & & $\mathrm{x}$ & & $\mathrm{x}$ \\
\hline Take actions where necessary & & $\mathrm{x}$ & & $\mathrm{x}$ \\
\hline Review the competency if necessary & & $\mathrm{x}$ & & $\mathrm{x}$ \\
\hline
\end{tabular}

* Assessment Measures

In 2014, the university updated its GE curriculum to include areas of understanding which comprise four key ILOs that include a total of ten competencies. To assess these competencies, the VALUE rubric (Rhodes, 2009) was modified and applied across campus. This activity demonstrated the institution's commitment to ensuring that ILOs were achieved and that a degree granted from the university reflected high quality, a goal of the Multi-State Collaborative (MSC). This effort also responded to a widespread objective of using standardized testing in higher education. Most importantly, the ILOs assessment using a modified VALUE rubric provided the opportunity for faculty to have conversations about improvement of student learning outcomes (Wehlburg, Carnahan \& Rhodes, 2017).

4.1.2. ILOs assessment results

In AY 2017-2018, faculty collected students' artifacts from 230 sections aligned with Competency 1 (written communication), Competency 2 (oral communication), Competency 3 (quantitative literacy) and Competency 5 (managing information) more than half of the artifacts $(57 \%, 2,858)$ had been assessed by the instructors and loaded into the AMS. For the remaining $43 \%$ of artifacts, in some cases, faculty did not collect the data and in others, improvements in the assignments are needed so faculty can independently score the artifacts. Of the four competencies, Competency 3 received the highest response rate $(76 \%)$ and Competency 2 received the lowest response rate $(42 \%)$.

Table 2. Modified VALUE Rubric Response Rate 2017-2018

\begin{tabular}{lccccc}
\hline & $\begin{array}{c}\text { Written } \\
\text { Communication }\end{array}$ & $\begin{array}{c}\text { Oral } \\
\text { Communication }\end{array}$ & $\begin{array}{c}\text { Quantitative } \\
\text { Literacy }\end{array}$ & $\begin{array}{c}\text { Managing } \\
\text { Information }\end{array}$ & Total \\
\hline Total Students & 1610 & 828 & 1218 & 1330 & 4986 \\
Total Reponses & 752 & 350 & 924 & 832 & 2858 \\
\% of Response & $\mathbf{4 7 \%}$ & $\mathbf{4 2 \%}$ & $\mathbf{7 6 \%}$ & $\mathbf{6 3 \%}$ & $\mathbf{5 7 \%}$ \\
\hline
\end{tabular}

On average, $98 \%$ of freshmen met the requirement, scoring 1 or above in the modified VALUE rubric. Of the four competencies, oral communication and quantitative literacy scored a higher average of 2.4.

In Spring 2018, the University Assessment Coordinator prepared the university ILOs assessment report and shared it with several groups and committees across campus including Academic Council, department chairs, GE Committee, Faculty Senate University Assessment Council (FSUAC) and the faculty group that has been involved in the data collection of Modified VALUE rubrics. The purpose of the council meeting was to review assessment results and discuss the strategies to improve next year's response rates using the Modified VALUE rubrics. The 
discussion with the GE Committee was to facilitate their use of assessment results in the recertification process. In addition to aggregated assessment results for the whole university, the assessment coordinator provided the assessment report by competency. The faculty meetings were set up by the vice provost, university assessment coordinator, and GE coordinator to share the results and ask for feedback. One of the key and critical components of the assessment process remains a challenge: documenting actions for improvement from each competency.

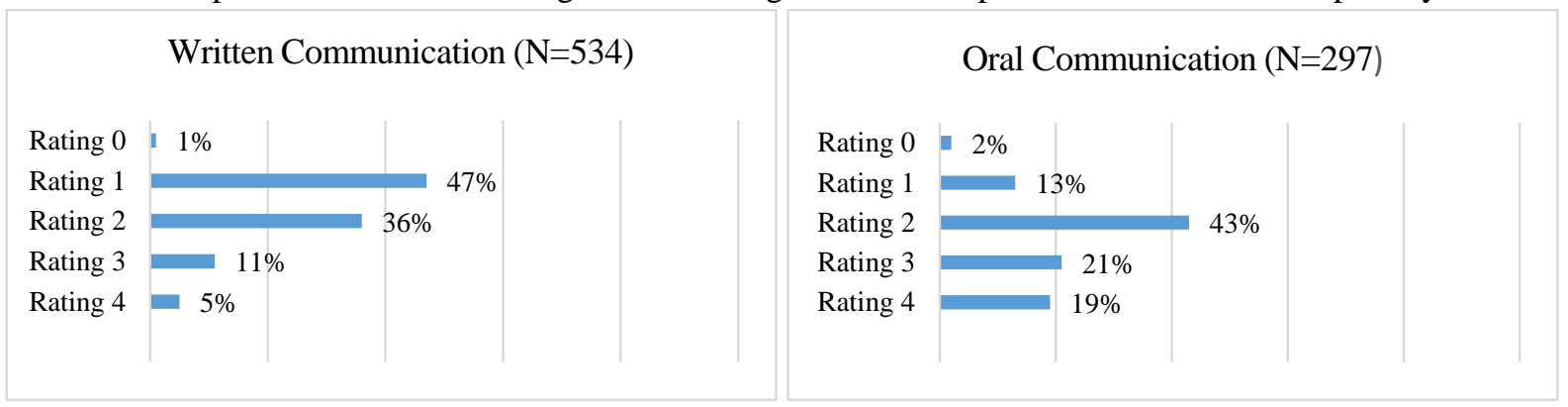

Figure 2. Four competencies of ILOs Assessment Result

\subsubsection{Challenges encountered}

In two years since IQA of ILOs was implemented, the university encountered several challenges. The first challenge was the technology. Although it provided the ability to collect and analyze a great deal of information, some faculty had issues in the implementation such as being unable to create a link in the LMS, inappropriate data display or issues with artifact submission by students. The second challenge was the faculty interpretation of the modified VALUE rubrics. Although training about the modified VALUE rubrics was done before the data collection, some faculty had a hard time determining and assigning the scores from the rubric to their own assignment, especially when the student received a low score in the rubric and still got the A grade in their course. The third challenge was the participation rate across the institution. Although more than 2,000 artifacts were collected, it accounted for only $57 \%$ of the population. Some faculty decided not to submit any artifacts from their course in the system. Some had challenges separating out the individual artifacts. The fourth challenge was the lack of infrastructure to engage faculty who are directly involved in the ILOs assessment process to discuss results of student learning and to identity changes for quality improvement. Finally, university ILOs assessment results relied on one artifact or one assignment; therefore, the reliability of results was sometimes questioned, creating a barrier in making appropriate changes for improvement.

\subsubsection{Close the loop efforts}

Based on the challenges encountered in AY18-19, the university prioritized three solutions to facilitate closing the loop in the ILOs assessment process. University administrators acknowledged the critical value of faculty coming together to discuss student learning and pedagogy to identify opportunities to better support teaching and learning in GE courses. Therefore, the first improvement was to create a time and place for faculty to engage in deep and meaningful conversations about student learning and effective teaching. To facilitate this strategy, the university established lead faculty for each competency. The major responsibilities of these faculty members were to lead the discussion of the assessment results within their group, and document the feedback and recommendations to improve the assessment process and possible actions for improvement. The university provided a template with key components in the assessment cycle to facilitate the documentation of meeting minutes. The second adjustment was to improve the validity and reliability of student artifacts. The university provided training and workshops on "assignment design" and calibration workshop series facilitated by university assessment coordinator and external presenters. These lead faculty members served as facilitators to promote professional development opportunities and to coordinate faculty meetings to discuss and review actions taken in response to learning outcomes data. The third improvement was to embed assessment data into GE recertification. Previously, the GE committee ensured the course learning outcomes and course assignments aligned with GE competencies. This adjustment ensured that student performance meets the expectation of course learning outcomes and the course assignment.

\subsection{Discussion}

\subsubsection{The alignment of IQA process with previous research}

The university IQA process of ILOs was established to meet the demand of external stakeholders for more accountability in student learning. Therefore, the consistent IQA process would also benefit the accreditation-related efforts (Council for Higher Education Accreditation, n.d). This aligned with the university goal to create processes and 
strategies that made assessment practice and assessment transparent to all faculty. This practice followed the VALUE rubric to capture the $21^{\text {st }}$ century skills that all graduates were expected to demonstrate by their graduation. The university IQA implementation process - from two-year timeline, data collection, multiple assessment-related faculty workshops throughout the academic year, and the use of central AMS system to store and analyze assessment data followed the lessons learned from other universities in AAC\&U report (McConnell \& Rhodes, 2017; Martin, 2017).

Another effort of this IQA process was the widespread faculty engagement in the assessment process from assignment design to pedagogy, data collection, and discussion of assessment results (Brow et al., 2018; Macdonald et al., 2014; Swarat \& Wrynn, 2017). Two features of this process, personnel work and technological tools, distributed the responsibility for assessment of SLOs so that no one person was solely responsible in the IQA process. Multiple coordinators at different levels (university, college, department, and competency) facilitated faculty engagement in meaningful discussion of assessment findings and regular conversations about teaching practices. Most important, faculty could experience assessment activities as opportunities for their own learning and professional growth when attending the annual training about teaching and learning improvement. At the same time, lead faculty served as the leaders in their group to facilitate closing the loop discussions (Pham \& Koch, 2019).

This IQA process embedded a meta-assessment that enabled faculty to evaluate their own practice (Schoepp \& Benson, 2016; Pham, 2020). After attending meetings to discuss assessment results within their competency, faculty were encouraged to run the report, watch a video on the assessment website on the strategies of interpreting assessment data, and then fill in the GE assessment self-reflection sheet (Appendix A). This process allowed faculty to determine the strengths and weaknesses of student learning for their own course, then decided what actions they could make for improvement. This meta-assessment was not intended to evaluate faculty assessment efforts but to assisted them in using assessment results to improve their own practices. Multiple minor but systematical changes implemented over time can produce substantive impact on teaching and learning (Stanny et al., 2015).

Certain short-term strategies can move the IQA process forward. The first is to improve the alignment of SLOs at different levels (university, GE, and academic programs) to facilitate skill-based assessment at the senior level. Seniorlevel data not only ensures students who are nearing graduation have had opportunities to improve, practice, and develop skills related to the competencies, but allows the university to provide evidence of student growth over time. The University Assessment Committee will work with academic programs to ensure that appropriate skills are embedded in their program learning outcomes. A pilot can be implemented in which faculty teaching capstone courses will use the modified VALUE rubric to assess student performance. Faculty can use one capstone assignment to assess multiple skills. Faculty will decide which skills the capstone should align with and select the appropriate rubric(s). The pilot of capstone assessment will facilitate university plans to implement assessment across the entire academic time frame for students. The second strategy is to improve the validity and reliability of assessment results by encouraging more meaningful actions for improvement. The university can build an inter-rater reliability system that includes a second faculty member assessing sample artifacts of the five competencies. Finally, the university can consider having an ILOs Assessment Committee to discuss and continue to improve the IQA process of ILOs. Right now, the GE assessment activities are initiated and overseen at the academic administrative level. To transition the assessment functions to the GE committee or to form a committee specifically addressing ILOs assessment, the university needs to transfer some of the ownership to faculty and help with dissemination of information. This committee can also offer support with inter-rater reliability as well as documentation of discussions and recommendations for annual ILOs assessment reports. 4.2.2. Implications for Vietnam HEIs

All HEIs are under pressure to provide evidence of student performance to internal and external stakeholders. Therefore, the experience from a case study in United States can be applicable for Vietnam HEIs to provide evidence of learning performance to MOET and for accreditation. First, Vietnam HEIs should look at the institution's mission to set up appropriate ILOs for the first 60 credits in the first two years. For best practices, they could consult the list of $21^{\text {st }}$ century skills that AAC\&U developed and choose those that are necessary for the Vietnam context. Second, institutions should require that courses in the first two-year curriculum align with appropriate ILOs. To ensure the alignment, the course learning outcomes need to address the ILOs language in the course objectives. Third, Vietnam HEIs should choose a reliable assessment measures to collect data. The VALUE rubric is designed to move from standardized exam to authentic assessment, using the authentic students' artifacts to make improvement of student learning. Some U.S. HEIs use the available assessment rubric to collect data. Some adopt the language in the rubric. Others use the VALUE rubric as a framework to build their own rubric. Vietnam HEIs can similarly choose appropriate approaches. Researchers recommend using the available rubric then make changes later as necessary. 
Fourth, in order to implement IQA of ILOs successfully, one strategy to engage faculty is to provide guidance and understanding of the entire IQA process, why it is being undertaken, and what the outcomes of the process will be used for. Vietnam HEIs should consider providing professional development opportunities for faculty teaching the courses on how to design the assessment to align with the rubric, how to read, integrate and use the rubric to score students' assignment and how to provide consistent scoring across the courses. This is a very significant step to avoid the challenges in validity and reliability in the data collection. Figure 1 on IQA of ILOs process provides additional information on how Vietnam HEIs can share assessment results with multiple committees as a way to close the assessment loop for quality improvement of student learning. Lastly, Vietnam HEIs should consider having a metaassessment, assessing the assessment process in place such as peer review of assignment design to ensure the validity of the assignment, calibration to ensure the reliability of the students' scores across the multiple courses and survey on faculty perceptions about the IQA process.

\section{CONCLUSION}

This case study conducted at a U.S. comprehensive university provided a step-by-step guide to help other universities implement IQA of ILOs, beginning with a two-year timeline, appropriate assessment measures, ILOs data analysis and challenges encountered, as well as efforts to link assessment with continuous quality improvement. This case study built on previous research and a U.S initiative to integrate authentic assessment into the process. The results from this case study can be applicable to many other HEIs that want to provide evidence of student learning to stakeholders. In addition to sharing the stages in IQA of ILOs as well as the challenges, this case study provided detailed steps to discuss the assessment results with internal stakeholders to close the assessment loop. This step is often missing in the IQA process or is not conducted effectively. Most important, this case emphasizes the characteristic of institutional effectiveness that administrators used the ILOs assessment results in the resources allocation to improve the student learning institution wide.

To sustain the culture of continuous improvement, the university needs to maintain a long-term strategy. The first is to provide continuous professional development opportunities for GE faculty, especially the adjuncts. The university in this case study continues to offer incentive, such as research or scholarship opportunities, for faculty to determine whether the pedagogical changes they make in their course produce improvement in student learning. These efforts can lead to the creation of an assessment network where faculty can design and develop a common course-based assignment for courses. Another strategy to build the culture of assessment is to hold an annual teaching and learning fair, poster sections, workshops, or think tanks where faculty facilitate discussions on assessment results and implications. The major goal of these events is to enhance faculty understanding of the assessment process, facilitate the use of data, evaluate the assessment cycle, and determine whether the assessment process leads to real changes in student learning. The final strategy is to engage students in GE assessment process. Although the university administers the NSSE, it is not administered annually. To triangulate assessment data from both direct and indirect assessment measures, instructors can ask students to reflect in class and use that feedback for indirect authentic assessment evidence in addition to the student assignment artifacts (Hutchings, 2018). That feedback could include qualitative data which our process has not yet formally included.

\section{REFERENCES}

Asia-Pacific Quality Network (APQN) (2008). Higher education quality assurance principles for the Asia Pacific Region. Paper presented at the APQN, Shanghai.

Bassis, M. (2015). A Primer on the transformation of higher education in America. Retrieved from http://www.learningoutcomeassessment.org/documents/BassisPrimer.pdf

Brown, S., McGrevy, J., Berigan, N. (2018). Evidence-Informed improvement through collaborative professional integration. New Directions for Teaching and Learning, 155, 55-64. https://doi:10.1002/t1.20303

Bruce, R. T. (2018). Assessment in Action: Evidence-Based discussions about teaching, learning, and curriculum. New Directions for Teaching and Learning, 10(2), 1-7. https://doi.org/10.1002/tl.20260

Council for Higher Education Accreditation. (n.d.). Retrieved from https://www.chea.org/regional-accrediting-organizations

Creswell, J. W. (2014). Research design: Qualitative, quantitative, and mixed methods approach (4t ${ }^{\text {th }}$ ed.). Sage Publications, Thousand Oaks, CA.

Fletcher, L., Meyer, H., Anderson, P., Johnston, M. R. (2012). Faculty and students' conceptions of assessment in higher education. Higher Education, 64(1), 119-133. http://www.jstor.org/stable/41477923 
Hutchings, P. (2018). Helping students develop habits of reflection: What we can learn from the NILOA Assignment Library. Urbana, IL: University of Illinois and Indiana University, National Institute for Learning Outcomes Assessment (NILOA).

Jones, D. A. (2009). Higher education assessment - Who are we assessing, and for what purpose?. Retrieved from https://www.aacu.org/publications-research/periodicals/higher-education-assessment\%E2\%80\%94who-are-weassessing-and-what-purpose

Kuh, G. D., Ikenberry, S. O., Jankowski, N. A., Cain, T. R., Ewell, P. T., Hutching, P. \& Kinzie, J. (2015). Using evidence of student learning in improve higher education. San Francisco, CA: Jossey-Bass.

Le, C. L. (2020). Suggestions to improve the internal quality assurance system to meet the requirements of educational innovation. Conference proceeding: Quality assurance of higher education in the new context. VNUHCMC Publishing House, 72-89.

LEAP. (2018). Retrieved from https://www.aacu.org/leap

Macdonald, S., Williams, L., Lazowski, R., Horst, S., \& Barron, K. (2014). Faculty Attitudes toward General Education Assessment: A Qualitative Study about Their Motivation. Research \& Practice in Assessment.

Martin, M. (2017). Internal Quality Assurance: Enhancing higher education quality and graduate employability. UNESCO Publishing.

McConnell, K. D. \& Rhodes, T. L. (2017). On solid ground. Retrieved from https://www.aacu.org/OnSolidGroundVALUE

Merriam, S. (1998). Qualitative research and case study applications in education. Jossey-Bass Publications, San Francisco, CA.

Ministry of Education and Training (MOET) (2007). Decision No. 65/2007/QD-BGDDT promulgating regulations on standards for accreditation of universities.

Nelson, C. (2014). Assessing assessment. Retrieved from https://www.insidehighered.com/views/2014/11/24/essaycriticizes-state-assessment-movement-higher-education

Nguyen, T. (2012). Internal quality assurance in Vietnam higher education: the influence by international projects. Master thesis, University of Twente.

Nguyen, T. S. (2020). Application of information technology in the internal quality assessment of Ho Chi Minh City University of Technology. Conference proceeding: Quality assurance of higher education in the new context. VNU-HCMC Publishing House, 46-60.

Penn, J. D. (2011). The case for assessing complex general education student learning outcomes. New Directions for Institutional Research, 149, 5-14. https://doi.org/10.1002/ir.376

Pham, T. T. N. \& Koch, D. (2019). Closing the assessment loop in General Education Program. In Smith, M. Generally speaking: The impact of General Education on Student Learning in the $21^{\text {st }}$ Century. Myers Education Press.

Pham, T. T. N. (2020). Multiple meta-assessment measures of a quality process: toward institutional effectiveness. Quality Assurance in Education, 28(2),123-136. https://doi.org/10.1108/qae-08-2019-0080

Pham, X. T. (2013). Higher education quality assurance in Vietnam. Paper presented at the 2013 AQAN seminar and roundtable meeting.

Rhodes, T. (2009). Assessing outcomes and improving achievement: Tips and tools for using the rubrics. Washington, DC: Association of American Colleges and Universities.

Schoepp, K., \& Benson, S. (2016). Meta-assessment: Assessing the learning outcomes assessment program. Innovative Higher Education, 41(4), 287-301. https://doi.org/10.1007/s10755-015-9347-2

Stanny, C., Gonzalez, M. \& McGowan, B. (2015). Assessing the culture of teaching and learning through a syllabus review. Assessment \& Evaluation in Higher Education, 40(7), 898-913.

Swarat, S. \& Wrynn, A. M. (2017). Assessment with Benefits: Faculty engagement and community building through GE assessment. (Assessment in Practice). Urbana, IL: National Institute for Learning Outcomes Assessment (NILOA).

Van, T. T. \& Phan, H. P. (2020). Internal Quality Assurance of Initial Teacher Education Programs in Vietnam: A Descriptive Study. International Journal of Innovation. Creativity and Change, 12(10), 226-244.

Vo, D. N. \& Ngo, K. C. (2020). Internal quality assessment of training program in accordance with the internal standards at Ho Chi Minh City University of Technology, Vietnam National University, Ho Chi Minh City. Ho Chi Minh City University of Technology, 25-45.

Wehlburg, C., Carnahan, J. \& Rhodes, T. (2017). Multi-State collaborative to advance quality student learning. Retrieved from https://www.aacu.org/sites/default/files/MSC_Demonstration_Year.pdf

Yin, R. K. (1994). Case study research: Design and methods. Sage Publications, Thousand Oaks, CA. 